\title{
4D Active and passive thermography measurement system using a KUKA KR16 robot and time-of-flight imaging
}

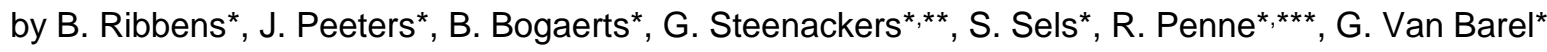 \\ * University of Antwerp, Faculty of Applied engineering, Op3Mech, Salesianenlaan 90, B-2660 Antwerp, \\ Belgium,bart.ribbens@uantwerpen.be \\ ** Vrije Universiteit Brussel (VUB), Acoustics \& Vibration Research Group (AVRG), B-1000 Brussels, Belgium \\ *** University of Antwerp, Department of Mathematics, Middelheimlaan 1, B-2020 Antwerp, Belgium
}

\begin{abstract}
Despite the fact that most thermography research is still based on 2D imaging techniques, researchers are investigating the true power and calibration of thermal imaging in three dimensions $[1,2]$. In our research multi-camera systems are built up around time-of-flight sensors [3-5], used to capture the geometric shape of the test object. The measurement setup and methodology we propose includes an industrial KUKA KR-16W robot which makes it possible to scan the object from multiple viewpoints and adds time as an extra $4^{\text {th }}$ dimension during the passive thermography experiments. This automated system helps to compensate the influence of directional emissivity [6].
\end{abstract}

Keywords: 4D thermography, Robotics, Time-of-flight, Data fusion

\section{Introduction}

In the last 20 years, non-destructive research is increasing with the rise of the carbon fabricated composite structures [1]. Beside the traditional methods, recent developments are made in more advanced techniques such as shearography, ultrasonic polar scanning and active thermography to perform more quantitative measurements [7]-[10]. Recent research goes further than just defect detection. Currently there is a need for methods to quantitatively estimate failure size, failure location and material properties [10], [11], which results in large, time consuming and complex calculations [10].

Active thermography can be seen as a fast surface method which is used extensively for qualitative purposes or initial inspections of high risk spots with respect to material failure [11]. But for quantitative purposes, it has several disadvantages in comparison of non-optical techniques. In this contribution we will discuss geometrical corrections and the use of Time of Flight (ToF) cameras to measure complex geometries.

While most thermographic NDT research is performed on flat test samples such as flat bottom hole plates, general thermographic applications can benefit from 3D measurements. Such a 3D thermographic measurement combines a geometrical three-dimensional scan of the object (laser scanning, structured-light, time-of-flight, etc.) and a thermal image of the scene. In this case the pixels of the thermal imaging device are mapped onto the measured real world coordinates. We chose to use a time-of-flight sensor because it can be mounted onto a robot and compared to laser triangulation only one image is needed instead of multiple line scans. It gives us immediately a full-field 3D image while a point laser scanner has to scan each point individually [1].

A first advantage is that large complex structures can be measured and visualized by combining several 3D thermographic scans. Secondly, if the orientation of the thermal imaging device is known with respect to the object, the influence of directional emissivity can be characterized and compared with numerical experiments [6]. The proposed setup is expected to allow compensation for this directional emissivity during experiments using the geometric information. Finally, because the measured temperatures correspond with known locations on the surface of the object, a validation with numerical simulations based on CAD data becomes much easier.

To automate the measurements, a thermal camera, a depth sensor, an RGB camera and a heat source are mounted onto a 6-axis industrial robot. The robot moves constantly around the object under test and creates a multidimensional point cloud. Each point contains the Euclidian location ( $x, y, z)$, a temperature and a color (RGB). This multi-spectral 3D point cloud is later referred to as a smart data cloud. An extra advantage of this automated setup is that there is no operator required in the room and therefore external influences are minimized. Repeatability is also guaranteed since the measurement locations are known and the operator can even choose the ideal measurement orientations in advance [12]. A third advantage is that there is only one IR imaging device needed to view measure the object from different orientations. During active thermography the heat source mounted on the robot is used to generate heat pulses or specific heat sequences.

This paper is structured as follows. Section 2 motivates for this research. Section 3 and Error! Reference source not found., describes our measurement setup and methodology. The calibration procedure is clarified together with its corresponding measurement errors in section 4. Finally, the results are shown, followed by our planned future work. 
First, a motivation for this research is given, proceeded by a description of our measurement setup and methodology. Further, we explain our calibration procedure together with its corresponding measurement errors. Finally, the results are shown, followed by our planned future work.

\section{Motivation for the present study}

There are several issues that motivate the present research:

- From an industrial perspective it is mandatory to register the local temperatures of a test subject in three dimensions instead of two dimensions to make sure that the temperatures correspond with a real location on the surface of the test sample.

- While line or point scanning techniques need a lot of different and time-consuming scans, time-of-flight imaging is a fast full-field measurement technique.

- Scanning large surfaces and performing automatic calibration benefits from using a robot.

- Validating numerical simulations of heat transfers over time can take some time depending on the test object, for example to investigate the heat propagation of a heat sink. Also if thermal information of different sides of the object is desirable, a multi-camera setup is needed. Both these issues can be overcome by using a robot as a multi-axis manipulator. In this case the robot is continuously scanning measuring the object from different viewpoints using only one infrared camera in combination with a 3D scanning device.

- Thermal imaging is often performed in a conditioned environment and the presence of an operator can be excluded by the use of a robot.

- The accurate movements and known locations of the manipulator simplify the merging of the smart data clouds corresponding with each individual viewpoint.

- Combining 3D, IR and RGB information enables a representation in which the user can recognize each part by its color and shape and see the local temperature of this particular part.

\section{Measurement setup}

Two major categories of thermal imaging cameras currently exist: the photon detectors and the thermal detectors [9]. Photon detectors transform the radiation directly in an electric signal due to the absorption of photons. This delivers a time accurate measurement with a stable integration time. Drawbacks of these cameras are that they need to be cooled to cryogenic temperatures and the cost price is also an issue. Further on, they have a high accuracy but only on a narrow bandwidth. The alternative is a microbolometer camera which transforms in a first stage the incident radiation into heat by absorption whereby the bolometer temperature changes. In a second stage the temperature difference is related to a difference in electric resistivity of the detector material [9]. This results in a time constant of approximated 4-12ms [13], [14]. This thermal constant delivers a constant delay for each pixel. The temperature read-out is highly influenced by the ambient conditions and the temperature of the sensor housing [9]. The readout is working following the rolling shutter principle [9] which results in a time delay between the pixel at the top-left and the pixel at the bottom-right. The optical behavior of the IR radiation is the same as other electromechanical radiation. Only the used materials are dependent of the transmission bands [11]. In this research a microbolometer camera is used but in near future a cooled camera will be mounted onto the robot to increase the thermal accuracy of the system.

To capture three-dimensional thermal data in an automated way, multiple detectors are mounted onto a 6-axis industrial robot (KUKA KR-16W) as shown in Fig. 1. The main component is the high resolution uncooled microbolometer camera Xenics Gobi-640-GigE. Due to its Ethernet connectivity the camera can be operated from a long distance, which is ideal when using a robot. To measure the 3D geometry of test object, a MESA imaging SwissRanger 4000 time-offlight camera is used. This camera is also Ethernet based and both devices can be combined using a gigabit router. The setup is completed by an IDS imaging uEye UI124xSE RGB camera operated through usb 2.0. For active thermography testing a halogen lamp is mounted underneath the camera base plate as shown in Fig. 2. During the calibration procedure the light is controlled by a DMX4ALL USB-DMX Mini Interface connected to a Velleman DMX relais. When calibrating the infrared camera, a second halogen bulb is added to illuminate the checkerboard pattern from a different location, and that light is linked to the same DMX channel. For the active thermography we use a National Instruments $\mathrm{DAQ}$ device in combination with an amplifier to control the mounted halogen lamp.

Our robot is controlled from within MATLAB using an intermediate layer called OPC Unified Architecture (OPC $U A)$. This makes it possible to send coordinates to the robot and capture our image data using the same software (MATLAB). By using this method, we are able to synchronize the whole image acquisition system while gathering images from multiple viewpoints without the need of extra imaging devices. An extra advantage is that the tool coordinates are known accurately which serve as a first estimation of the camera poses. To calculate the exact camera poses, a handeye calibration is needed. 


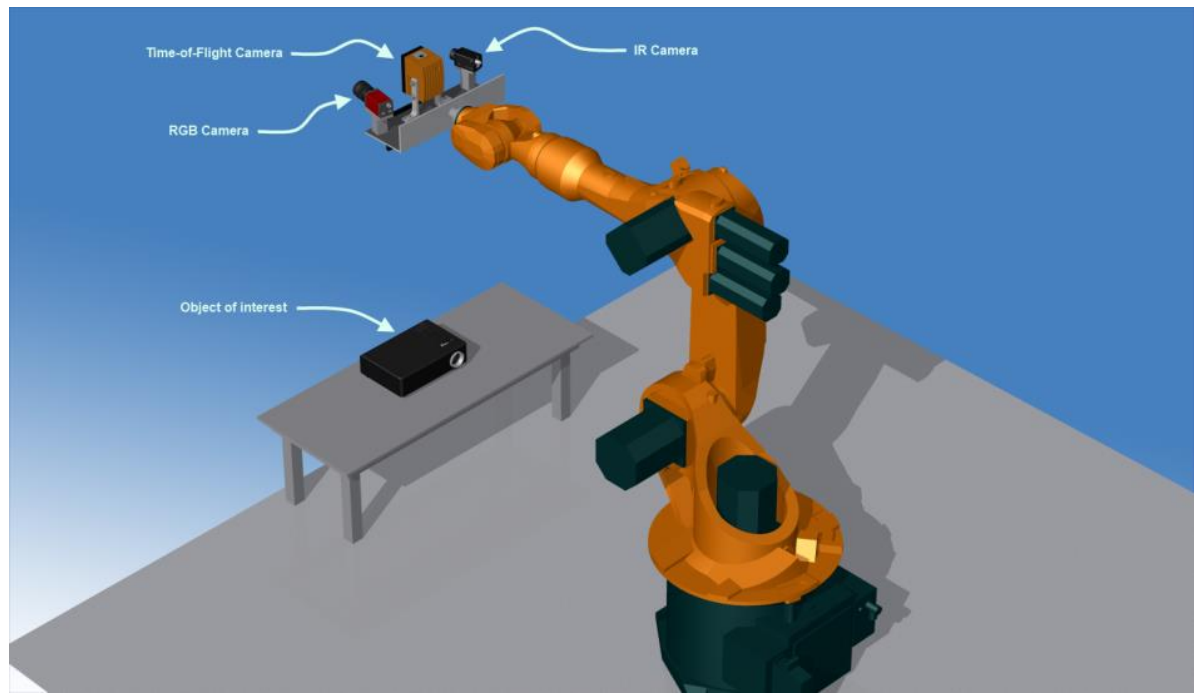

Fig. 1. Robotic 3D thermal imaging system. The image shows the thermal, time-of-flight and RGB camera, the light source for active thermography and the object of interest.

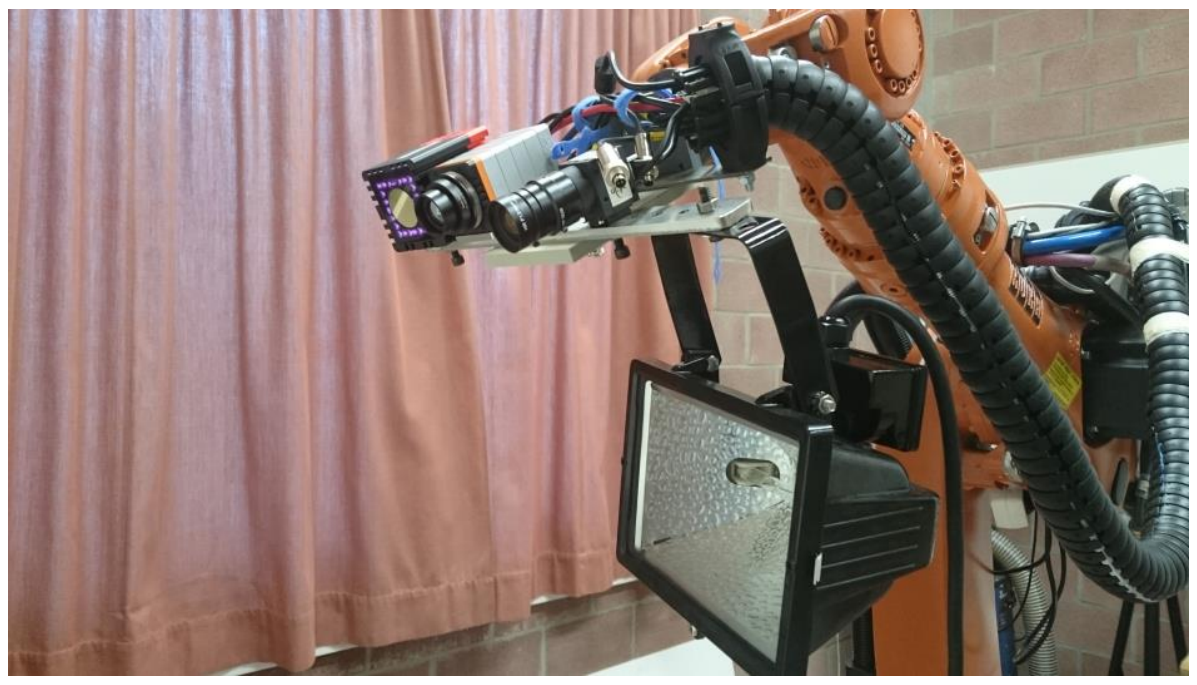

Fig. 2. Three-dimensional thermal imaging system existing of a MESA SR4000 time-of-flight camera (left), a XENICS Gobi 640 thermal camera (middle), a CMOS uEye UI124xSE RGB camera and a 500 Watt halogen light.

\section{Calibration}

This research became possible by the development of new calibration techniques for time-of-flight cameras and the combination of different state-of-the-art sensors [3-5, 7]. Although we presented a novel geometrical calibration technique earlier for 3D IR imaging [14], this paper is mainly based on the camera calibration toolbox that was integrated in MATLAB since 2015 for the convenience of the reader.

A first step in the calibration procedure is to obtain the intrinsic calibration parameters of the infrared camera. These intrinsic parameters consist of nonlinear lens distortion coefficients and the intrinsic matrix of the camera.

The goal of the calibration procedure is to obtain the rigid transformation between the ToF camera and the IR camera. In this procedure a checkerboard is used. This is because a checkerboard is often used in camera calibration and is easy to produce. In this setting there are 3 coordinate frames. These frames are the IR camera frame, the ToF frame and the checkerboard frame. Given the intrinsic matrix of the IR camera and the image pixels of the checkerboard in the IR image it is relatively straightforward to compute the transformation between the checkerboard and the IR camera [15], [16]. For this approach to work it is crucial to find the checkerboard corner points in the IR image. In our setup this is achieved by turning on a 500 Watt light bulb for 5 seconds. The difference in emissivity between the black and white checkers leaves a distinguishable temperature difference which allows the detection of the checker corners 


\subsection{1/qirt.2016.106}

when a custom thresholding algorithm is used. Given this transformation, the location of the checkerboard corners is known in the frame of the infrared camera. These coordinates are denoted by $\left[\begin{array}{lll}X_{i}^{I R} & Y_{i}^{I R} & Z_{i}^{I R}\end{array}\right]^{T}$.

The straightforward way of determining these checkerboard coordinates in the reference frame of the ToF camera is by detecting the checkerboard in the luminance image of this camera. The ToF camera also gives $X Y Z$ coordinates for each luminance pixel. These values aren't reliable because the checkerboard corners are black white transitions. 3D measurements of a ToF camera at black white transitions are less reliable. For this reason, a plane was fitted through the XYZ values of the white checkers of the checkerboard, these values are reliable (MATLAB does have a built in function pcfitplane which uses ransac). The $X Y Z$ values of the checkerboard corners are restored by calculating the intersection of the rays through the checkerboard pixels of the luminance image and the 3D plane of the checkerboard. These XYZ values are the locations of the checkerboard corners in the frame of the ToF camera and are denoted by $\left[\begin{array}{lll}X_{i}^{\text {TOF }} & Y_{i}^{\text {TOF }} & Z_{i}^{\text {TOF }}\end{array}\right]^{T}$.

Given these labeled corresponding points in two coordinate frames, it is straightforward to calculate the rigid transformation between the two reference frames as described in [17]. The corresponding points of multiple checkerboard can be added in the least squares approximation of this transformation. In our approach we used this transformation as a starting point for a nonlinear optimization of this transformation.

When this transformation is known we can assign a temperature (color) to each pixel of the ToF camera which is seen by the IR camera. The parameters that are necessary for this operation are the rotation $(R)$ and translation (t) between both sensors, the intrinsic matrix $(\mathrm{K})$ of the IR camera and the measured $\mathrm{XYZ}$ value of each pixel of the ToF camera $\left(\left[\begin{array}{lll}X_{\tilde{i}} & Y_{\tilde{i}} & Z_{i}\end{array}\right]^{T}\right)$. With these parameters it is possible to calculate the corresponding pixel value of the IR image for each pixel of the ToF camera as follows:

$$
\left[\begin{array}{l}
u \\
v \\
W
\end{array}\right]=K\left[\begin{array}{ll}
R & t \\
0 & 1
\end{array}\right]\left[\begin{array}{c}
X_{i} \\
Y_{1} \\
Z_{1} \\
1
\end{array}\right]
$$

\section{Measurement errors of smart data clouds}

While creating a three-dimensional heat image, multiple inaccuracies arise. First, there are measurement errors due to the time-of-flight principle. In this paper we use a MESA SwissRanger SR4000 camera. The heart of this camera is an advanced sensor technology employing the time-of-flight distance measurement principle where infrared light from the camera's internal lighting source is reflected by objects in the scene and travels back to the camera where its precise time of arrival is measured independently by each of tens of thousands of sensor pixels. The SR4000 camera that was used in this research is a phase shift amplitude modulation measurement device that modulates its illumination LEDs (Light Emitting Diodes) at $30 \mathrm{MHz}$ and its imaging sensor measures the phase of the returned modulated signal at each pixel. The distance at each pixel is determined as a fraction of the one full cycle of the modulated signal [18]. Because the signal (phase) is influenced by the reflection coefficient and scattering of the material it is bouncing back from, inaccuracies occur. This causes the effect that black checkerboard squares are measured at a different depth as the white squares of the checkerboard pattern. Also the shape of an object can distort the measured image due to erroneous reflections [19]. Therefore, it would be interesting to have a technique to evaluate the precision of a time-of-flight sensor. However, during the last decade several research groups tried to compare some time-of-flight cameras $[1,2,3]$ but there is no official standard regarding three-dimensional measurements [4]. This makes it impossible for manufacturers or other research groups to evaluate their ToF cameras objectively by benchmarking them with other products. The research results of the National Research Council Canada (NRC) [4, 5, 10] are applicable to sheet-of-light measurements, but the typical problems that arise using time-of-flight measurements are neglected. Manufacturers often use the depth precision of $+-1 \mathrm{~cm} / \mathrm{m}$ to show the quality of their device.

Second, measurement errors originate from combining the thermal image with the $X Y Z$ coordinates of the ToF sensor. Both sensors are first calibrated intrinsically and afterwards extrinsically, but since these calibrations are based on corner detection of checkerboard patterns, the defined parameters are estimated values with their own error. To calculate the relative pose between the sensors (extrinsic calibration), a homography is needed. To calculate this homography, a minimal of 4 point correspondences is needed ( 3 non-collinear points!). This homography is calculated with respect to the calibration plane that contains the checkerboard pattern and its accuracy is related to the accuracy of the detected checkerboard points.

At this moment we are unaware of a method to calculate the global error magnitude that represents both the errors of the time-of-flight measurement and the data fusion procedure.

\section{Results}

To validate the calibration and the measurement procedure of the system, a three-dimensional scan of four billiard balls on a wooden plane is executed as shown in Fig. 3. Three balls are cooled down to about thirteen degrees, while the other was at room temperature as shown in Fig. 4. 


\subsection{1/qirt.2016.106}

Together with the XYZ-coordinates, a thermal image and RGB image is recorded. By using the intrinsic camera matrix of the IR camera and the rotation and translation matrix that describe the position of the IR camera with respect to the ToF camera, the thermal data is mapped onto the measured XYZ-coordinates. The mapping of the RGB coordinates onto the $\mathrm{XYZ}$-coordinates is similar to the IR mapping, but these results are not shown in this proceeding.

Next to the $X Y Z$-coordinates, the Mesa imaging SR4000 ToF camera gives a luminance image which is shown in Fig. 5. This 3D representation of the measured scene also makes it easier to understand the thermal 3D representation shown in Fig. 6. Fig. 6 shows that the calibration between the IR and ToF camera works very good regarding the limited precision of the ToF sensor $(1 \mathrm{~cm} / \mathrm{m})$. Therefore, this research demonstrates that a printed checkerboard can be used in combination with an extra light source to calibrate such a system.

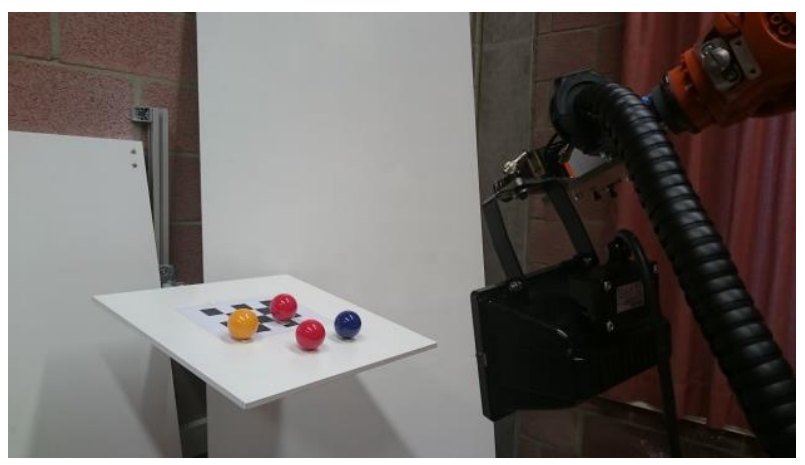

Fig. 3. Four billiard balls of which 3 are cooled down to +-13 degrees Celsius.

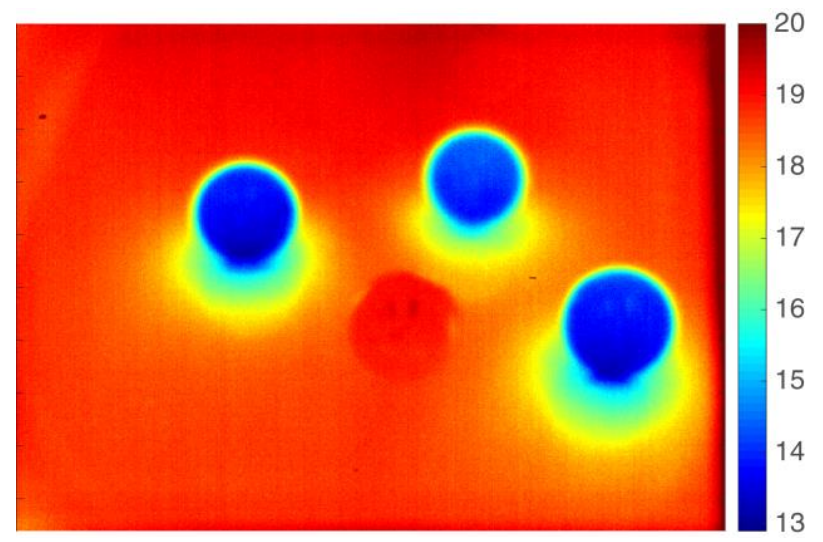

Fig. 4: Thermal image of four billiard balls. Three are cooled down while one is at room temperature. 


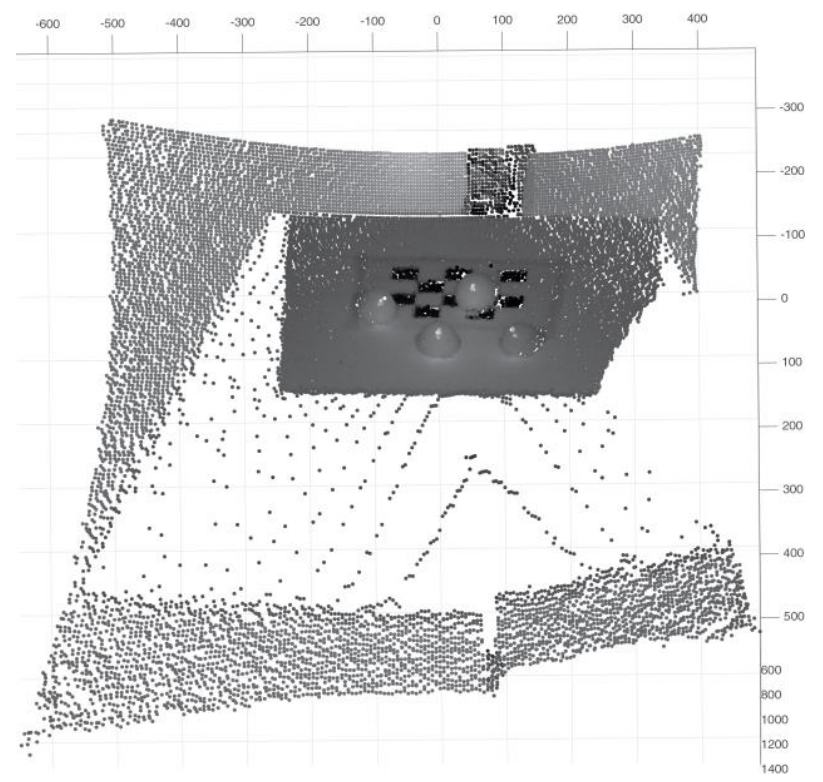

Fig. 5: Luminance image of the MESA SwissRanger 4000 camera visualized in three dimensions.

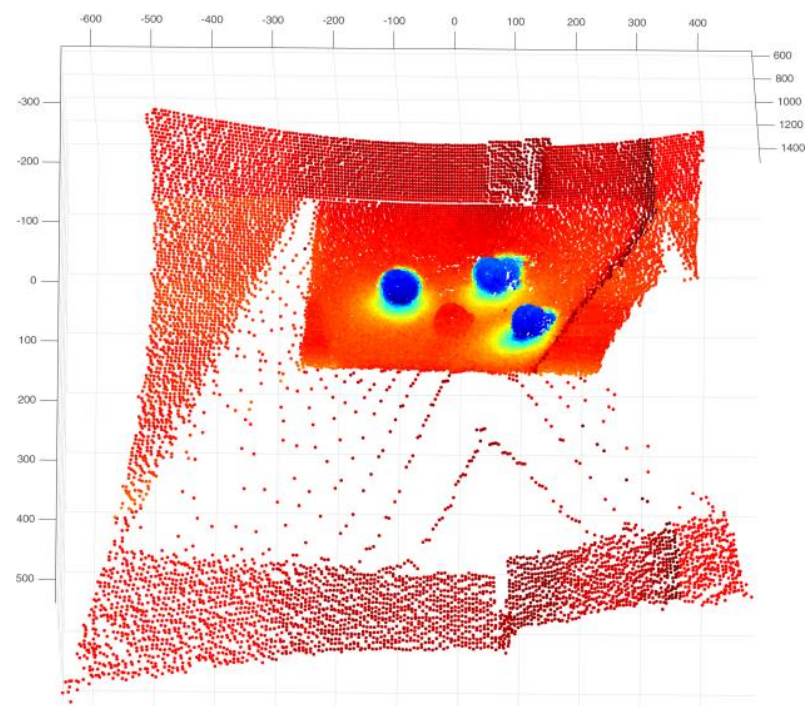

Fig. 6: This image shows the measured point cloud including the temperature mapping we performed. You can see that three balls have a much lower temperature than the environment.

\section{Conclusions}

In this paper, an automatic calibration procedure for three-dimensional thermal imaging based on a time-of-flight camera and an industrial robot is elaborated. Furthermore, we showed that 3D thermal imaging is possible by combining a ToF sensor with a microbolometer infrared camera. When investigating an object for a certain period of time (4D), we can evaluate the local temperature changes and later compare them to numerical simulations.

\section{Future work}

To improve the quality of the three-dimensional thermal point clouds, a more accurate geometrical scanning technique and a better thermal camera is needed. Therefore, in near future we will combine a projection profilometry system [21] with a cooled infrared camera. A full-field projection profilometry system can easily give 3D points in space with a precision of $0.2 \mathrm{~mm}$ [22], which is a huge difference with the $1 \mathrm{~cm}$ precision that is obtained using time-of-flight imaging sensors. Also because of the high spatial resolution of the projection based technique, the precision of the 
geometrical calibration will improve which results in a better correspondence between the IR data and the Euclidian coordinates.

As soon as we have a more accurate 3D thermal imaging system we will further investigate the compensation of the directional emissivity by using geometrical data as shown in [6]. Scans from different viewpoints will be combined in a dense smart data cloud with $\mathrm{XYZ}$ coordinates, heat values and color information. By using the link between the experimental data and the numerical model [14], a thorough evaluation of the simulation results can be executed.

Finally, it is shown in this research that the need increases to develop objective criteria for three-dimensional (time-of-flight) measurements, but in particular for multispectral 3D measurements (smart data clouds) on a scale of one millimeters up to one meter. Guidelines should be written to objectively compare different systems.

\section{ACKNOWLEDGMENTS}

This research has been funded by the University of Antwerp (Stim-KP 31128) and the government agency Flanders Innovation \& Entrepreneurship (VLAIO) by the support to the TETRA project 'Smart data clouds' with project number 140336.

\section{REFERENCES}

[1] M. A. Akhloufi and B. Verney, "Multimodal Registration and Fusion for 3D Thermal Imaging," Math. Probl. Eng., vol. 2015, no. Article ID 450101, p. 14, 2015.

[2] J. Rangel, S. Soldan, and a Kroll, "3D Thermal Imaging: Fusion of Thermography and Depth Cameras," Conf. Quant. InfraRed Thermogr., 2014.

[3] L. Mertens, R. Penne, and B. Ribbens, "Time of flight cameras (3D vision)," in Recent advances in topography research, Nova Science Publishers, 2013, pp. 353-417.

[4] R. Penne, C. Raposo, L. Mertens, B. Ribbens, and H. Araujo, "Investigating new calibration methods without feature detection for ToF cameras," Image Vis. Comput.

[5] R. Penne, B. Ribbens, and L. Mertens, "An Incremental Procedure for the Lateral Calibration of a Time-of-Flight Camera by One Image of a Flat Surface," Int. J. Comput. Vis., pp. 1-11, 2014.

[6] J. Peeters, B. Ribbens, J. J. J. Dirckx, and G. Steenackers, "Numerical estimation of directional emissivity: experimental validation with thermographic measurements," Int. J. Heat Mass Transf.

[7] G. De Angelis, M. Meo, D. P. Almond, S. G. Pickering, and S. L. Angioni, "A new technique to detect defect size and depth in composite structures using digital shearography and unconstrained optimization," NDT E Int., vol. 45, no. 1, pp. 91-96, Jan. 2012.

[8] M. Kersemans, "Combined Experimental-Numerical Study to the Ultrasonic Polar Scan for Inspection and Characterization of (Damaged) Anisotropic Materials." UGent, 2014.

[9] M. Vollmer and K.-P. Moellmann, Infrared thermal imaging: fundamentals, research and applications. 2010.

[10] V. Vavilov, "Thermal NDT - Historical milestones , state -of-the-art and trends," in QIRT2014 Conférence, 2014, p. 1.

[11] X. Maldague, Theory and practice of infrared thermography for nondestructive testing. Quebec: Wiley, 2001.

[12] S. Dutta and T. Schmidt, "New concept for higher Robot position accuracy during thermography measurement to be implemented with the existing prototype automated thermography end-effector utilising an industrial robot and laser system."

[13] D. Piatti, "Time-of-Flight cameras: tests, calibration and multi-frame registration for automatic 3D object reconstruction Dario Piatti," 2010.

[14] J. Peeters, I. Coudron, L. Mertens, J. Dirckx, and G. Steenackers, "Defining geometrical position information of a thermal camera and object curvatures using thermography," in OPTICAL MEASUREMENT TECHNIQUES FOR STRUCTURES \& SYSTEMS III, 2016, pp. 233-242.

[15] Z. Zhang, "A flexible new technique for camera calibration," IEEE Trans. Pattern Anal. Mach. Intell., vol. 22, no. 11, pp. 1330-1334, Nov. 2000.

[16] J. Y. Bouguet, "Camera calibration toolbox for Matlab." 2008.

[17] D. W. Eggert, a. Lorusso, and R. B. Fisher, "Estimating 3-D rigid body transformations: a comparison of four major algorithms," Mach. Vis. Appl., vol. 9, no. 5-6, pp. 272-290, 1997.

[18] F. Chiabrando, R. Chiabrando, D. Piatti, and F. Rinaudo, "Sensors for 3D Imaging: Metric Evaluation and Calibration of a CCD/CMOS Time-of-Flight Camera," Sensors (Basel)., vol. 9, no. 12, pp. 10080-10096, Dec. 2009. 
[19] A. Bhandari, A. Kadambi, R. Whyte, C. Barsi, M. Feigin, A. Dorrington, and R. Raskar, "Resolving multipath interference in time-of-flight imaging via modulation frequency diversity and sparse regularization," Opt. Lett., vol. 39, no. 6, pp. 1705-1708, 2014.

[20] Beraldin, J.-Angelo, Mackinnon, David, and Cournoyer, Luc, "Metrological characterization of 3D imaging systems: progress report on standards developments," International Congress of Metrology. p. 13003, 2015.

[21] B. Ribbens, V. A. Jacobs, C. Vuye, J. A. N. Buytaert, J. J. J. Dirckx, and S. Vanlanduit, "High-resolution temporal profilometry using Fourier estimation," in Recent advances in topography research, J. A. N. Buytaert, Ed. New York: Nova Science, 2013, pp. 61-108.

[22] S. S. Gorthi and P. Rastogi, "Fringe projection techniques: Whither we are?," Opt. Lasers Eng., vol. 48, no. 2, pp. 133-140, Feb. 2010. 